\title{
Recent Results on the Anomalous X-ray Pulsars
}

\author{
S. Mereghetti ${ }^{\mathrm{a}}$, L. Stella ${ }^{b}$ and G.L. Israel ${ }^{\mathrm{b}}$

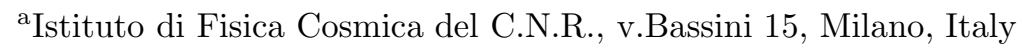 \\ ${ }^{\text {b}}$ Osservatorio Astronomico di Roma, v. dell'Osservatorio 2, Monteporzio Catone, Italy
}

The "Anomalous X-ray Pulsars" (AXP) are a small group of X-ray pulsars characterized by periods in the $\sim 5-10$ s range and by the absence of massive companion stars. There are now 7 possible members of this class of objects. We review recent observational results on their X-ray spectra, spin period evolution, and searches for orbital motion and discuss the implications for possible models.

\section{THE ACCRETING X-RAY PULSARS}

There is compelling evidence that most of the $\gtrsim 50$ accreting pulsars in $\mathrm{X}$-ray binaries have massive companion stars. They are therefore classified as High Mass X-ray Binaries (HMXRB). In most cases this is based on the observation of their optical counterparts. Ten X-ray pulsars are optically identified with OB supergiant stars, while Be companions have been found for $\sim 20$ pulsars. Many of the optically unidentified X-ray pulsars are inferred to be HMXRB, because they share the properties of the $\mathrm{Be} /$ neutron star systems (hard spectrum, transient behaviour, often with a recurrence equal to the orbital period).

The four X-ray pulsars optically identified with low mass stars (Her X-1, GX1+4, GRO J174428 and $4 \mathrm{U}$ 1626-67) form a very inhomogenous group, each source having its own peculiar properties [1,2].

In the last few years there has been growing evidence that a group of pulsars, clearly not belonging to the HMXRB class, possess the following prominent common properties [3]:

- their optical counterparts are very faint, implying that they cannot have massive companions

- their spin periods are distributed in a narrow range ( $5-10 \mathrm{~s})$, contrary to those of HMXRB that cover a much larger interval

- they have very soft $\mathrm{X}$-ray spectra (with the exception of $4 \mathrm{U}$ 1626-67)

- their X-ray luminosity is relatively low $\left(10^{35}\right.$. $10^{36} \mathrm{erg} \mathrm{s}^{-1}$ ) compared to that of HMXRB pul- sars

- their X-ray flux shows little variability on timescales from months to years (they are not transient systems)

- they have a relatively stable spin period evolution, with long intervals of nearly constant spindown

- a few of them are possibly associated to supernova remnants.

These objects have been called to in different ways by various authors: " 6 second pulsars" [何, braking pulsars [5], very low mass binary pulsars [3], anomalous X-ray pulsars (AXP). In the following we shall adopt the latter name.

\section{AXP INVENTORY}

The 5 sources originally considered [3] in the AXP group are : 1E 2259+586, 1E 1048.1-5937, $4 \mathrm{U} 1626-67,4 \mathrm{U}$ 0142+61, and RXJ 1838.4-0301.

Thanks to recent ASCA discoveries, two new possible members have been proposed. An 11 s periodicity has been found [6] in the source 1RXS J170849.0-400910 first discovered during the ROSAT All Sky Survey. Its very soft spectrum is similar to that of the AXP. Nothing is yet known on its optical counterparts. The second possible AXP is the unresolved $\mathrm{X}$-ray source 1E 1841-045 discovered with the Einstein satellite [7] near the geometrical center of the young $(\sim 2000$ yr $)$ supernova remnant Kes 73 and recently found to be pulsed at $11.8 \mathrm{~s}[8]$.

Optical observations of the field of RXJ 1838.4- 
Table 1

AXP with two component spectra

\begin{tabular}{|c|c|c|c|c|c|}
\hline & $\begin{array}{r}k T \\
(\mathrm{keV})\end{array}$ & $\begin{array}{r}R_{b b} \\
(\mathrm{~km})\end{array}$ & $L_{b b} / L_{t o t}$ & $\begin{array}{r}\text { power law } \\
\text { photon index }\end{array}$ & ref. \\
\hline$\overline{4 \mathrm{U} 0142+61}$ & 0.39 & $2.4 d_{1 k p c}$ & $\overline{0.4}$ & 3.7 & 11. \\
\hline $1 \mathrm{E} 2259+586$ & 0.44 & $3.3 d_{4 k p c}$ & 0.4 & 3.9 & 12 \\
\hline 1E 1048.1-5937 & 0.64 & $0.6 d_{3 k p c}$ & 0.5 & 2.5 & 13 \\
\hline 1RXS J170849.0-400910 & 0.41 & $4.5 d_{5 k p c}$ & 0.2 & 2.9 & 6 \\
\hline $4 \mathrm{U} 1626-67$ & $0.3-0.6$ & $3-5 d_{3 k p c}$ & $0.1-0.2$ & 0.8 & (15), 16 \\
\hline
\end{tabular}

0301 revealed the presence of a main sequence K5 star with $\mathrm{V} \sim 14.5$ [9]. This star could be responsible for the observed $\mathrm{X}$-ray flux, since the implied X-ray to optical flux ratio is compatible with the level of coronal emission expected in late type stars. In the lack of an independent confirmation of the $5.45 \mathrm{~s}$ pulsation 10] the inclusion of RXJ 1838.4-0301 in the AXP group should be considered tentative.

Another questionable AXP is $4 \mathrm{U}$ 1626-67. Though originally included in the AXP group [3], various authors pointed out its different nature [4,5], on the basis of its hard spectrum and of the fact that it is the only source of this group with an identified optical counterpart and a well established binary nature.

\section{X-RAY SPECTRA}

Recent ASCA 11 and BeppoSAX 12,13 observations have shown that the spectra of $1 \mathrm{E} 2259+586,1 \mathrm{E} 1048.1-5937$ and $4 \mathrm{U} 0142+61$ cannot be described by single power laws. Their spectra are well fitted by the combination of a blackbody-like component with $\mathrm{kT} \sim 0.5 \mathrm{keV}$ and a steep power law with photon index $\alpha \sim 3-4$ (see Table 1). The same two-component spectrum is compatible with the ASCA data for 1RXS J170849.0-400910, although a simple power law gives a formally acceptable fit [6].

The spectrum of 1E 1841-045 [8] can be fit with a highly absorbed, soft power law $(\alpha \sim 3)$. A blackbody component would be difficult to detect due to the high column density $\left(\mathrm{N}_{H} \sim 210^{22}\right.$ $\mathrm{cm}^{-2}$ ). RXJ 1838.4-0301 has been observed so far only in the ROSAT band (0.1-2.4 keV), where it has a very soft $(\alpha \sim 3)$ though poorly con- strained spectrum [10.

The spectrum of $4 \mathrm{U} 1626-67$, a flat power law followed by an exponential cut-off above $\sim 20$ $\mathrm{keV}$ 14, is more typical of accreting pulsars in HMXRB. However, the presence of a soft blackbody component has been reported by several authors [15, 16]. A complex spectral feature around $1 \mathrm{keV}$, interpreted as emission from hydrogen-like neon [15], might indicate that the companion star is an He burning star.

\section{PERIOD EVOLUTION}

Spin-down on a timescale of $\sim 10^{4}-10^{5}$ years is one of the distinctive properties of AXP (see Table 2). Variations in the spin-down rate have been observed in the sources for which many period measurements are available 17, 12,13. In the case of $1 \mathrm{E} 2259+586$, these fluctuations are consistent with the torque noise measured in accreting systems [18] (that is several orders of magnitude greater than that of radio pulsars).

$4 \mathrm{U}$ 1626-67 again stands out for its different properties. In fact this source has been nearly steadily spinning-up for more than a decade after its discovery. In 1990 it underwent a rapid torque reversal and, since then, it has been spinning down at a rate nearly equal to the previous spin-up rate 19 .

Nothing is known yet on the period evolution of 1RXS J170849.0-400910 and RXJ 1838.4-0301.

\section{SEARCHES FOR ORBITAL MOTION}

No periodic X-ray flux modulations such as dips or eclipses have been detected in AXP. In the lack of optical identifications, the search for Doppler delays in the pulse arrival times is the 


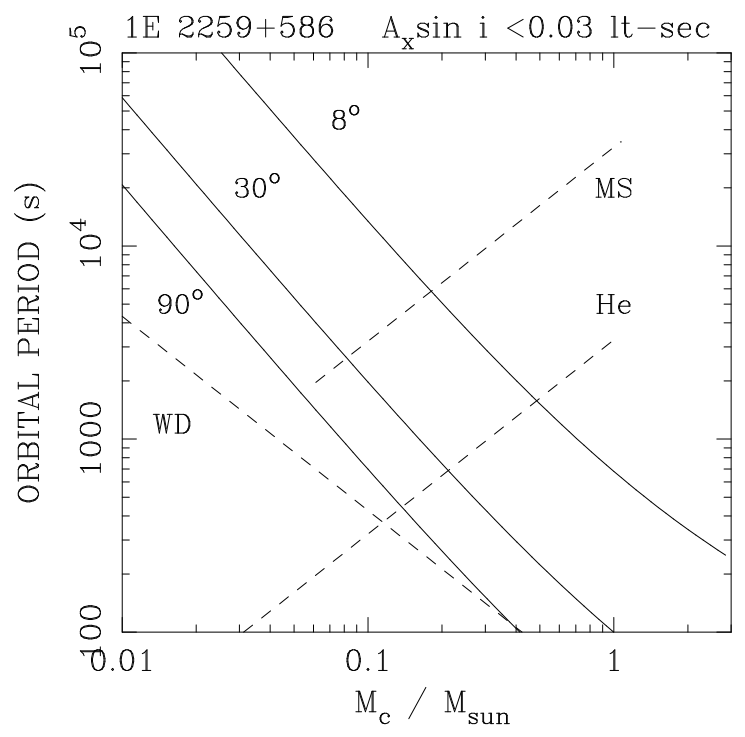

Figure 1. Orbital onstraints from the $a_{x} \sin i$ limit for $1 \mathrm{E} 2259+586$.

only way to assess their possible binary nature. This has been done with different satellites for the sources listed in Table 2, where the upper limits on $a_{x} \sin i$ are summarized.

The most constraining limits are those recently derived with the RossiXTE satellite for 1E 1048.1-5937 and 1E 2259+586 [20], as well as the value of 8 light-ms for $4 \mathrm{U}$ 1626-67 [21].

In this source, in addition to the $\mathrm{X}$-ray periodicity at $7.7 \mathrm{~s}$, a pulsation at a slightly lower frequency is present in the optical band [22]. This is probably due to reprocessing of the $\mathrm{X}$-ray pulses occurring near the companion star, and the difference of the two periodicities can be explained with an orbital period of $41.4 \mathrm{~min}$. Since this orbital period has not been confirmed with independent methods, we give in Table 2 also the limits on $a_{x} \sin i$ for other possible values of $P_{\text {orb }}$.

\section{DISCUSSION}

Though the precise nature of the low mass companion of $4 \mathrm{U} 1626-67$ is still unclear, it is well established that this source consists of a neutron star accreting in a binary system. Considering the differences discussed above between $4 \mathrm{U}$ 1626-67 and the other sources, it is not straightforward to assume a similar model for all the AXP. Though an optical counterpart as faint as that of $4 \mathrm{U} 1626-$ $67(\mathrm{Mv} \sim 5)$ cannot be ruled out at the highly absorbed positions of some of the other AXP, a more serious inconsistency is the difference in spectral shape. It is unlikely that this results from a different inclination of the line of sight, considering the similarities among the spectra of HMXRB pulsars that likely encompass systems at different orbital inclinations.

The $P$ and $\dot{P}$ values measured in AXP imply a rotational energy loss of the order of $10^{32}-10^{33}$ $\mathrm{erg} \mathrm{s}^{-1}$ (in the case of neutron stars with $I=10^{45}$ $\mathrm{g} \mathrm{cm}^{2}$ ), too small to power the observed luminosities. A spinning down, isolated white dwarf would provide a larger rotational energy loss thanks to its higher momentum of inertia. Such a model was originally proposed [23,24] for 1E $2259+586$. However this possibility has been ruled out by the $\dot{P}$ variations observed in the last few years (see section 3). Indeed the observed variations in $\dot{P}$, correlated with luminosity changes and occasionally implying short spin-up intervals, are a strong indication that accretion is occurring in these systems [17, 18].

It has been proposed [4] that the AXP (with the exception of $4 \mathrm{U}$ 1626-67) are the descendant of Thorne-Zytkow objects and consist of isolated neutron stars accreting from residual disks. The blackbody component observed in some AXP has been interpreted as evidence for quasi-spherical accretion onto an isolated neutron star formed after common envelope evolution and spiral-in of a massive X-ray binary [11,5]. In this case, the accretion flow results from the remaining part of the massive star envelope and is thought to consist of two components: a low-angular momentum component, giving rise to the blackbody emission from a considerable fraction of the neutron star surface, and a high-angular momentum one, forming an accretion disk responsible for the power-law emission and for the long term spindown evolution.

Though this is certainly a very interesting interpretation to be investigated with future observations, a more standard binary scenario is also 
Table 2

AXP Timing properties

\begin{tabular}{|c|c|c|c|c|c|}
\hline & $\begin{array}{r}P \\
\text { (sec) }\end{array}$ & $\begin{array}{r}P / \dot{P} \\
\text { (years) }\end{array}$ & $\begin{array}{r}a_{x} \sin i \\
\text { (light-sec) }\end{array}$ & Range of $P_{o r b}$ & ref. \\
\hline$\overline{4 U 0142+61}$ & 8.69 & $(4-15) 10^{4}$ & $0.37(99 \%)$ & $430 \mathrm{~s}-12 \mathrm{hr}$ & 28 \\
\hline $1 \mathrm{E} 2259+586$ & 6.98 & $410^{5}$ & $0.03(99 \%)$ & 194 s - 1 day & 20 \\
\hline 1E 1048.1-5937 & 6.45 & $(5-14) 10^{3}$ & $0.06(99 \%)$ & $200 \mathrm{~s}-1$ day & 20 \\
\hline \multirow[t]{5}{*}{$4 \mathrm{U} 1626-67$} & 7.66 & $510^{3}$ & $0.008(3 \sigma)$ & $P_{\text {orb }}=42 \mathrm{~min}$ & 21 \\
\hline & & & $0.013(3 \sigma)$ & $600 \mathrm{~s}-10 \mathrm{hr}$ & 27 \\
\hline & & & $0.1(2 \sigma)$ & $1 d-2 d$ & (26] \\
\hline & & & $0.06(2 \sigma)$ & $2 d-60 d$ & 19] \\
\hline & & & $0.15(2 \sigma)$ & $60 d-900 d$ & 19] \\
\hline 1RXSJ170849-400910 & 11.00 & - & - & & [6] \\
\hline $1 \mathrm{E} 1841-045$ & 11.77 & $810^{3}$ & - & & $\sqrt{8}$ \\
\hline RXJ 1838.4-0301 & 5.45 & - & - & & [10] \\
\hline
\end{tabular}

possible. In fact, the presence of very low mass white dwarfs or He-burning companions cannot be ruled out, despite the limits on $a_{x} \sin i$.

In the case of $1 \mathrm{E} 2259+586$ this is illustrated in figure 1 , where the limits on orbital period, $P_{\text {orb }}$, versus mass of the companion, $M_{c}$, are plotted assuming three different values for the unknown inclination angle. The dashed lines indicate the positions of Roche-lobe filling companions under the assumption of conservative mass transfer driven by angular momentum losses due only to gravitational radiation [25]. They refer to the cases of a main sequence, a He burning star and a fully degenerate hydrogen white dwarf. Values of $P_{\text {orb }}$ and $M_{c}$ below the corresponding dashed line are excluded, while those above the lines require accretion through stellar wind.

It is clear that, unless all these systems are nearly face-on, the most likely mass donors are white dwarfs and helium stars with masses below a few tenths of solar masses. In the latter case, the mass transfer is most likely through a stellar wind, since the accretion expected from a Rochelobe filling He-star would produce a far greater luminosity than the observed one.

\section{REFERENCES}

1. Nagase F., PASJ, 41, 1 (1989).

2. Kouveliotou C., et al., Nature, 379, 799 (1996).
3. Mereghetti S. \& Stella L., ApJ, 442, L20 (1995).

4. van Paradijs J., et al., A\&A, 299, L41 (1995).

5. Ghosh P., et al., ApJ, 478, 713 (1997).

6. Sugizaki M., et al., PASJ, 49, L25 (1997).

7. Kriss G.A., et al., ApJ, 288, 703 (1985).

8. Vasisht G. \& Gotthelf E.V., ApJ, 486, L129 (1997).

9. Mereghetti S., et al., A\&A, 321, 835 (1997).

10. Schwentker O., A\&A, 286, L47 (1994).

11. White N.E., et al., ApJ, 463, L83 (1996).

12. Parmar A., et al., A\&A, in press.

13. Oosterbroek T., et al., A\&A, submitted.

14. Pravdo S.H., et al., ApJ, 231, 912 (1979).

15. Angelini L. et al., ApJ, 449, 41 (1995).

16. Orlandini M., et al. , these proceedings.

17. Mereghetti S., ApJ, 455, 598 (1996).

18. Baykal A. \& Swank J.H., ApJ, 460, 470 (1996).

19. Chakrabarty D., et al., ApJ, 474, 414 (1997).

20. Mereghetti S., Israel G.L. \& and Stella L., MNRAS, in press.

21. Shinoda K., et al., PASJ, 42, L27 (1990).

22. Middleditch J.,et al, ApJ, 244, 1001 (1981).

23. Morini M., et al., ApJ, 333, 777 (1988).

24. Paczynski B., ApJ, 365, L9 (1990).

25. Verbunt F. \& van den Heuvel E.P.J., in $X$-ray Binaries, Cambridge Univ. Press, 457 (1995).

26. Joss P.C., et al., ApJ, 221, 645 (1978).

27. Levine A., et al., ApJ, 327, 732 (1988).

28. Israel G.L., et al., ApJ, 433, L25 (1994). 